\title{
Optical manifestations of domains with constant topological charge density
}

\author{
Evan Stewart and Kirill Tuchin $\odot$ \\ Department of Physics and Astronomy, Iowa State University, Ames, Iowa 50011, USA
}

(Received 26 June 2019; published 6 September 2019)

\begin{abstract}
Domains of finite topological charge density can exist in chiral materials and chiral matter. Spatial and temporal variation of the average topological charge density, represented by the $\theta$ field, induces anomalous currents that are responsible for the chiral magnetic effect, the anomalous Hall effect, and other phenomena that are intimately related to the chiral anomaly. We consider domains with constant average topological charge density. We argue that even though the Maxwell equations in the bulk are not altered, the chiral anomaly manifests itself by the way of the boundary conditions. This is illustrated by several examples. The first example deals with the refraction of plane electromagnetic waves on a surface of a constant $\theta$ domain. We derive the modified Fresnel equations and discuss the effect of the chiral anomaly on the amplitude and polarization of the reflected and transmitted waves. In particular, we argue that the Brewster's angle is sensitive to the value of $\theta$. In the second example we compute the spectrum of the transition radiation at high frequencies and show that it is enhanced at finite $\theta$.
\end{abstract}

DOI: 10.1103/PhysRevResearch.1.023005

\section{INTRODUCTION}

The chiral anomaly [1,2] plays an important role in materials containing chiral fermions. At finite topological charge density they exhibit a number of novel $P$ - and $C P$-odd electromagnetic effects [3]. The topologically nontrivial background has different sources: In hot nuclear matter it is created by the random sphaleron-mediated transitions between different QCD vacua [4,5], in Weyl and Dirac semimetals it emerges in external parallel electric and magnetic fields [6], and in the cosmological models it is due to the presence of the axion field [7].

The topological charge density $q(x)$ is a microscopic quantity that has a complicated spacetime structure. However, in many applications one is only interested in the macroscopic properties of materials. The macroscopic theory emerges upon averaging the microscopic quantities over the small domains that contain a macroscopic number of particles. The macroscopic quantity that we employ to describe the topological charge density is the $\theta$ field defined as $\theta(x)=-\langle q(x)\rangle M^{-4}$, where $M$ is a phenomenological parameter of mass-dimension one. Interaction of the electromagnetic field with the domains of finite $\theta$ can be described by adding to the QED Lagrangian the axion-photon coupling term [8-10]

$$
\mathcal{L}_{A}=-\frac{c_{A}}{4} \theta F_{\mu \nu} \tilde{F}^{\mu \nu}
$$

where $c_{A}$ is the QED anomaly coefficient $[1,2]$. Since $\theta$ depends on $x$, Eq. (1) cannot be rewritten as a total derivative and removed from the Lagrangian. Instead, it contributes the

Published by the American Physical Society under the terms of the Creative Commons Attribution 4.0 International license. Further distribution of this work must maintain attribution to the author(s) and the published article's title, journal citation, and DOI. spatial and the temporal derivatives of $\theta$ to the modified Maxwell equations,

$$
\begin{aligned}
\partial_{\mu}\left(F^{\mu \nu}+c_{A} \tilde{F}^{\mu \nu} \theta\right) & =j^{\nu}, \\
\partial_{\mu} \tilde{F}^{\mu \nu} & =0,
\end{aligned}
$$

where $\tilde{F}^{\mu \nu}$ is a dual field.

We assume that the topological charge density is induced by the external sources, so that $\theta$ is an external nondynamical field. ${ }^{1}$ Moreover, we assume that $\theta$ is a slowly varying function of space and time so that at the leading order in derivatives we can write $\theta(x) \approx \theta(0)+x \cdot \partial \theta(0)$. Its time derivative can then be interpreted as the axial chemical potential [11-13], while its spatial derivatives (times $c_{A}$ ) as the splitting of the Weyl nodes in momentum space [14]. In the infinite medium, i.e., far away from the boundaries, $\theta(0)$ plays no role as Eqs. (2) depend only on the derivatives $\partial \theta$. Most previous studies concentrated on the effect of the chiral anomaly on the electromagnetic field through these derivatives and found a plethora of novel effects, the most remarkable of which are the chiral magnetic effect (CME) [3,6,15-17] and the chiral instability of the electromagnetic field [18-20].

However, in finite media, such as the $C P$-odd domains in a quark-gluon plasma, boundaries do matter. For example, recently it was shown in Ref. [21] that the CME is generated even at $\operatorname{constant} \theta$ in a time-dependent external magnetic field. Moreover, if $\theta$ is indeed a slowly varying function in the bulk, then its discontinuity at the domain surface is the main source of the chiral effects. It is thus worthwhile to study electrodynamics in the presence of domains with constant $\theta$. This is the simplest realization of the chiral anomaly in

\footnotetext{
${ }^{1}$ In a fully dynamical theory one includes the kinetic and self-action terms of the $\theta$ field. This yields a macroscopic version of the axion electrodynamics.
} 
the electromagnetic theory: Even though $\theta$ drops out the anomalous Maxwell equations (2) in the bulk, it emerges in the boundary conditions on the domain surfaces [see Eqs. (4) below].

The main goal of this paper is to use the macroscopic electrodynamics with anomalous boundary conditions to study electromagnetic wave refraction and the transition radiation by constant $\theta$ domains. We argue that these processes can be used as effective experimental tools to investigate the chiral matter/materials.

The paper is organized as follows. We begin Sec. II by writing down the boundary conditions (4) for the electromagnetic fields at the boundary of a finite- $\theta$ domain. We then employ them to study the reflection and transmission of plane electromagnetic waves at the flat boundary. The result is the set of generalized Fresnel equations (10) for the transition and reflection amplitudes of various polarizations. Our main observation is that the $\theta$ domains are optically active even if the index of refraction is the same on the two sides of the boundary. In Sec. III we compute the Brewster's angle, i.e., the angle at which a plane wave linearly polarized in the plane of incidence is not reflected. We observe that it increases with the wave frequency in a way that strongly depends on $\theta$, as indicated in Fig. 2. In Sec. IV we consider the emission of the transition radiation by an ultrarelativistic electrically charged fermion crossing the boundary between the vacuum and the $\theta$ domain. This problem has been recently solved for inhomogeneous domains $(\partial \theta \neq 0)$ in Refs. [22,23]. Here, we compute the spectrum (26) of the radiated photons in the case of constant $\theta$ and argue that it is enhanced as compared with the conventional transition radiation. We draw conclusions in Sec. V.

\section{THE FRESNEL EQUATIONS FOR A CHIRAL DOMAIN}

We focus on linear, homogeneous, isotropic, electrically neutral, and nonmagnetic chiral matter with complex refractive index $n(\omega)$. The anomalous Maxwell equations (2), also referred to as the Maxwell-Chern-Simons equations, that describe the macroscopic electrodynamics of this matter can be written in three-dimensional form as

$$
\begin{aligned}
\nabla \cdot \boldsymbol{B} & =0, \\
\nabla \cdot(\boldsymbol{D}+\tilde{\theta} \boldsymbol{B}) & =0, \\
\nabla \times \boldsymbol{E} & =-\partial_{t} \boldsymbol{B}, \\
\nabla \times(\boldsymbol{B}-\tilde{\theta} \boldsymbol{E}) & =\partial_{t}(\boldsymbol{E}+\tilde{\theta} \boldsymbol{B}),
\end{aligned}
$$

where $\boldsymbol{D}$ is electric displacement and the pseudoscalar field $\tilde{\theta}=c_{A} \theta$ is proportional to the average topological charge density sourced by the non-Abelian fields or some other topological field configurations mentioned in the previous section. The boundary conditions on the domain wall can be obtained directly from Eqs. (3). Denoting by $\Delta$ the discontinuity of a field component across the domain wall, one obtains [10]

$$
\begin{aligned}
\Delta B_{\perp} & =0, \\
\Delta\left(E_{\perp}+\tilde{\theta} B_{\perp}\right) & =0, \\
\Delta \boldsymbol{E}_{\|} & =0, \\
\Delta\left(\boldsymbol{B}_{\|}-\tilde{\theta} \boldsymbol{E}_{\|}\right) & =0,
\end{aligned}
$$

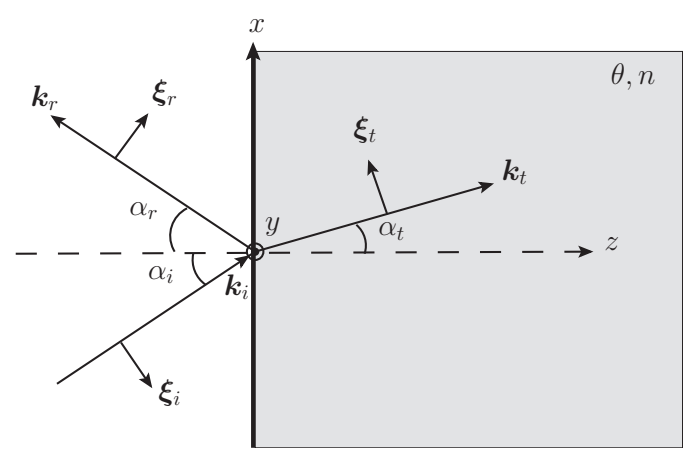

FIG. 1. Scattering of a plane circularly polarized electromagnetic wave off a semi-infinite domain (shaded area at $z \geqslant 0$ ) with index of refraction $n$ and constant $\theta$ field at an incident angle $\alpha_{i}$. The domain boundary is the $x y$ plane. The wave vectors lie in the $x z$ plane and the $y$ axis points at the reader. In the absorptive medium, the refraction index $n$ and the angle $\alpha_{t}$ are complex.

where $E_{\perp}, B_{\perp}$ and $\boldsymbol{E}_{\|}, \boldsymbol{B}_{\|}$are components of the electromagnetic field normal and tangential to the domain wall, respectively. We would like to use Eqs. (4) to compute how the refraction of electromagnetic waves is modified at finite constant $\theta$.

Consider the right-hand polarized plane monochromatic electromagnetic wave incident from vacuum $(z<0)$ on the flat boundary of a semi-infinite domain $(z>0)$ with constant $\theta$ field and constant $n$ (see Fig. 1). ${ }^{2}$ It is convenient to choose the radiation gauge in which the scalar potential vanishes and the vector potential is divergenceless. The vector potential outside the domain is then given by a superposition of the incident and reflected waves, while the vector potential inside the domain is given by the transmitted wave,

$$
\begin{aligned}
& \boldsymbol{A}_{1}=A_{i}^{+} \boldsymbol{\epsilon}_{i}^{+} e^{i \boldsymbol{k}_{i} \cdot \boldsymbol{r}}+A_{r}^{+} \boldsymbol{\epsilon}_{r}^{+} e^{i \boldsymbol{k}_{r} \cdot \boldsymbol{r}}+A_{r}^{-} \boldsymbol{\epsilon}_{r}^{-} e^{i \boldsymbol{k}_{r} \cdot \boldsymbol{r}}, \quad z<0, \\
& \boldsymbol{A}_{2}=A_{t}^{+} \boldsymbol{\epsilon}_{t}^{+} e^{i \boldsymbol{k}_{t} \cdot \boldsymbol{r}}+A_{t}^{-} \boldsymbol{\epsilon}_{t}^{-} e^{i \boldsymbol{k}_{t} \cdot \boldsymbol{r}}, \quad z>0,
\end{aligned}
$$

where the superscripts \pm refer to the right- and left-handed polarizations and we omitted the common time dependence factor $e^{-i \omega t}$. Since $\theta$ is constant at every point except at the boundary, it drops out the equations of motion (3). In particular, the dispersion relations are $k_{i}=k_{r}=\omega, k_{t}=n \omega$. Similarly, the continuity of the phase factors across the boundary requires that $k_{i x}=k_{r x}=k_{t x}$, giving the Snell's law $\sin \alpha_{i}=\sin \alpha_{r}=n \sin \alpha_{t}$. The effect of the finite $\theta$ appears only in the modified Fresnel equations.

In the reference frame depicted in Fig. 1, the polarization vectors are given by

$$
\boldsymbol{\epsilon}_{a}^{ \pm}=\frac{\hat{\boldsymbol{\xi}}_{a} \pm i \hat{\boldsymbol{y}}}{\sqrt{2}}, \quad a=i, r, t .
$$

The circularly polarized plane waves are eigenvectors of the curl operator by virtue of the identity

$$
i \hat{\boldsymbol{k}}_{a} \times \boldsymbol{\epsilon}_{a}^{ \pm}= \pm \boldsymbol{\epsilon}_{a}^{ \pm} .
$$

\footnotetext{
${ }^{2}$ A similar problem was considered by Hayata [24]. However, unlike the present study, he focused on the effects due to the inhomogeneity of $\theta$.
} 
Therefore, the magnetic field corresponding to (5a) and (5b) is given by

$$
\begin{aligned}
& \boldsymbol{B}_{1}=k_{i} A_{i}^{+} \boldsymbol{\epsilon}_{i}^{+} e^{i \boldsymbol{k}_{i} \cdot \boldsymbol{r}}+k_{r} A_{r}^{+} \boldsymbol{\epsilon}_{r}^{+} e^{i \boldsymbol{k}_{r} \cdot \boldsymbol{r}}-k_{r} A_{r}^{-} \boldsymbol{\epsilon}_{r}^{-} e^{i \boldsymbol{k}_{r} \cdot \boldsymbol{r}}, \quad z<0 \\
& \boldsymbol{B}_{2}=k_{t} A_{t}^{+} \boldsymbol{\epsilon}_{t}^{+} e^{i \boldsymbol{k}_{t} \cdot \boldsymbol{r}}-k_{t} A_{t}^{-} \boldsymbol{\epsilon}_{t}^{-} e^{i \boldsymbol{k}_{t} \cdot \boldsymbol{r}}, \quad z>0
\end{aligned}
$$

The electric field $\boldsymbol{E}=i \omega \boldsymbol{A}$ is simply proportional to the vector potential.

Projecting the boundary conditions (4c) and (4d) on the $x$ and $y$ axes yields

$$
\begin{aligned}
\cos \alpha_{i}\left(A_{i}^{+}-A_{r}^{+}-A_{r}^{-}\right)= & \cos \alpha_{t}\left(A_{t}^{+}+A_{t}^{-}\right), \\
A_{i}^{+}+A_{r}^{+}-A_{r}^{-}= & A_{t}^{+}-A_{t}^{-}, \\
\cos \alpha_{i}\left(A_{i}^{+}-A_{r}^{+}+A_{r}^{-}\right)= & n \cos \alpha_{t}\left(A_{t}^{+}-A_{t}^{-}\right) \\
& -i \tilde{\theta} \cos \alpha_{t}\left(A_{t}^{+}+A_{t}^{-}\right), \\
A_{i}^{+}+A_{r}^{+}+A_{r}^{-}= & n\left(A_{t}^{+}+A_{t}^{-}\right)-i \tilde{\theta}\left(A_{i}^{+}-A_{t}^{-}\right) .
\end{aligned}
$$

The other two boundary conditions (4b) and (4d) do not contain any new information. The solution to (9a)-(9d) reads

$$
A_{t}^{+}=\frac{\cos \alpha_{i}\left(\cos \alpha_{t}+\cos \alpha_{i}\right)(n+1+i \tilde{\theta})}{n\left(\cos ^{2} \alpha_{i}+\cos ^{2} \alpha_{t}\right)+\cos \alpha_{i} \cos \alpha_{t}\left(n^{2}+1+\tilde{\theta}^{2}\right)} A_{i}^{+},
$$

$$
A_{r}^{-}=\frac{\cos \alpha_{t} \cos \alpha_{i}\left[n^{2}-(1+i \tilde{\theta})^{2}\right]}{n\left(\cos ^{2} \alpha_{i}+\cos ^{2} \alpha_{t}\right)+\cos \alpha_{i} \cos \alpha_{t}\left(n^{2}+1+\tilde{\theta}^{2}\right)} A_{i}^{+}
$$

$$
A_{t}^{-}=\frac{\left(\cos \alpha_{t}-\cos \alpha_{i}\right)(n-1-i \tilde{\theta}) \cos \alpha_{i}}{n\left(\cos ^{2} \alpha_{i}+\cos ^{2} \alpha_{t}\right)+\cos \alpha_{i} \cos \alpha_{t}\left(n^{2}+1+\tilde{\theta}^{2}\right)} A_{i}^{+},
$$

$$
A_{r}^{+}=\frac{\left(\cos ^{2} \alpha_{i}-\cos ^{2} \alpha_{t}\right) n}{n\left(\cos ^{2} \alpha_{i}+\cos ^{2} \alpha_{t}\right)+\cos \alpha_{i} \cos \alpha_{t}\left(n^{2}+1+\tilde{\theta}^{2}\right)} A_{i}^{+},
$$

where $\cos \alpha_{t}=\sqrt{1-n^{-2} \sin ^{2} \alpha_{i}}$. In general, the polarization of the transmitted and reflected waves is elliptical, which is of course also true if $\theta=0$. However, at finite $\theta$ this holds even for the linearly polarized incident wave except in the special cases discussed below.

If the incident wave is left-hand polarized, the corresponding amplitudes can be obtained from (10) by noting that $\boldsymbol{E}$ and $\boldsymbol{B}$ are polar and axial vectors, respectively. Hence applying the parity transformation to the boundary conditions (4) is equivalent to replacing $\theta \rightarrow-\theta$ and $A_{a}^{ \pm} \rightarrow A_{a}^{\mp}(a=i, r, t)$ in the Fresnel equations (10).

Consider now several particular cases. In the limit $\theta=0$ one recovers the conventional Fresnel equations. In particular, for the normal incidence $\alpha_{i}=0$, the amplitudes are

$$
\begin{aligned}
& A_{t}^{+}=\frac{2}{n+1} A_{i}^{+}, \quad A_{r}^{-}=\frac{n-1}{n+1} A_{i}^{+}, \\
& A_{t}^{-}=A_{r}^{+}=0 \quad \text { if } \theta=\alpha_{i}=0 .
\end{aligned}
$$

If the media on the two sides of the boundary have the same index of refraction, but different values of $\theta$, e.g., the two $\theta$ vacua in $\mathrm{QCD}$, the boundary is still reflective. In particular, setting $n=1$ implies that $\alpha_{i}=\alpha_{r}=\alpha_{t}$ and the Fresnel equations (10) reduce to (at any $\alpha_{i}$ )

$$
\begin{aligned}
& A_{t}^{+}=\frac{2}{2-i \tilde{\theta}} A_{i}^{+}, \quad A_{r}^{-}=-\frac{i \tilde{\theta}}{2-i \tilde{\theta}} A_{i}^{+}, \\
& A_{t}^{-}=A_{r}^{+}=0 \quad \text { if } n=1 .
\end{aligned}
$$

We observe that (i) the amplitudes of the transmitted and reflected waves do not depend on the angle of incidence, (ii) a material with finite $\theta$ reflects electromagnetic waves with the effective index of refraction $n_{\text {eff }}=1-i \tilde{\theta}$ [as can be realized by comparing (11) and (12)], and (iii) the polarization of the transmitted wave is the same as the polarization of the incident wave. In other words, at $n=1, \theta \neq 0$ the circular polarization is preserved, in contrast to the $n>1, \theta=0$ case where the linear polarization is preserved. It follows from (12) that an electromagnetic wave traversing domains with different values of $\theta$, but the same refractive index, is reflected even at normal incidence. Experimentally, Eqs. (12) describe the scattering of high-frequency waves in which case the index of refraction is close to unity. We will return to this point in more detail in the following sections. In the Appendix we record the result for the refraction of a right-hand polarized wave normally incident at a thin film.

\section{BREWSTER'S ANGLE}

At a certain angle of incidence, known as the Brewster angle, there is no reflection of the incident wave component linearly polarized in the $x z$ plane. To determine the dependence of this angle on $\theta$, consider the linearly polarized incident wave. It can be written as a superposition of the right- and left-hand polarized waves as (throughout this section we omit the phase factors for brevity)

$$
\boldsymbol{A}_{i}=A_{i}^{+} \boldsymbol{\epsilon}_{i}^{+}+\mathcal{A}_{i}^{-} \boldsymbol{\epsilon}_{i}^{-}=\sqrt{2} A_{i}^{+} \begin{cases}\hat{\xi}_{i} & \text { if } A_{i}^{+}=\mathcal{A}_{i}^{-}, \\ \hat{\boldsymbol{y}} & \text { if } A_{i}^{+}=-\mathcal{A}_{i}^{-} .\end{cases}
$$

The calligraphic font refers to the amplitude of the left-hand polarized component to distinguish it from the results of the previous section [recall that Eqs. (10) are written for the

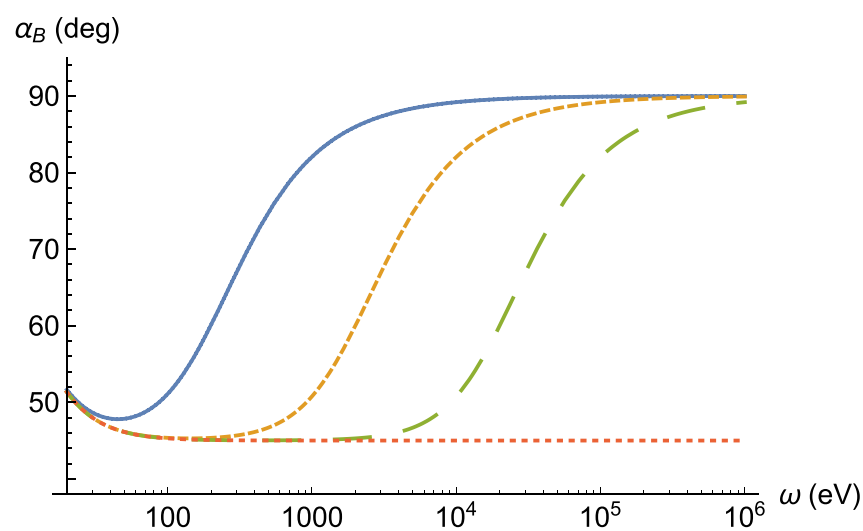

FIG. 2. Brewster's angle as a function of the wave frequency for a typical value of the plasma frequency $\omega_{\mathrm{pl}}=10 \mathrm{eV}$ at different values of $\tilde{\theta}=10^{-1}, 10^{-2}, 10^{-3}$ (left to right). The dotted line corresponds to $\theta=0$. 
right-hand polarized incident wave]. The vector potential of the reflected wave is

$$
\boldsymbol{A}_{r}=\left(A_{r}^{+}+\mathcal{A}_{r}^{+}\right) \boldsymbol{\epsilon}_{r}^{+}+\left(A_{r}^{-}+\mathcal{A}_{r}^{-}\right) \boldsymbol{\epsilon}_{r}^{-} .
$$

Substituting (10b) and (10d) into (14) and recalling that, as explained in the previous section, $\mathcal{A}_{r}^{ \pm}(\theta)=A_{r}^{\mp}(-\theta)$, we obtain for the incident wave polarized in the $x z$ plane (i.e., $\left.A_{i}^{+}=\mathcal{A}_{i}^{-}\right)$,

$$
\begin{aligned}
\boldsymbol{A}_{r}= & \sqrt{2} A_{i}^{+}\left\{\hat{\boldsymbol{\xi}}_{r} \frac{n\left(\cos ^{2} \alpha_{i}-\cos ^{2} \alpha_{t}\right)+\cos \alpha_{i} \cos \alpha_{t}\left(n^{2}-1+\tilde{\theta}^{2}\right)}{n\left(\cos ^{2} \alpha_{i}+\cos ^{2} \alpha_{t}\right)+\cos \alpha_{i} \cos \alpha_{t}\left(n^{2}-1+\tilde{\theta}^{2}\right)}\right. \\
& \left.+\hat{\boldsymbol{y}} \frac{2 i \tilde{\theta} \cos \alpha_{i} \cos \alpha_{t}}{n\left(\cos ^{2} \alpha_{i}+\cos ^{2} \alpha_{t}\right)+\cos \alpha_{i} \cos \alpha_{t}\left(n^{2}-1+\tilde{\theta}^{2}\right)}\right\} .
\end{aligned}
$$

The in-plane component of the reflected wave vanishes when $\alpha_{i}=\alpha_{B}$ where

$$
\tan ^{2} \alpha_{B}=\frac{\left(n^{2}-1+\tilde{\theta}^{2}\right)\left(n^{2}-1+\tilde{\theta}^{2}+\sqrt{\left(n^{2}+1\right)^{2}+\tilde{\theta}^{2}\left[\tilde{\theta}^{2}+2\left(n^{2}-1\right)\right]}\right)}{2\left(n^{2}-1\right)} .
$$

In Fig. 2 we plotted $\alpha_{B}(\omega)$ for several values of $\tilde{\theta}$. One can see that the Brewster's angle is quite sensitive to $\tilde{\theta}$.

There are two limiting cases: If $|\tilde{\theta}| \ll 1$ at fixed $n$, then

$$
\tan \alpha_{B} \approx n+\frac{n^{3}}{n^{4}-1} \tilde{\theta}^{2} .
$$

The first term on the right-hand side is the conventional result for $\theta=0$. The second term is a correction that implies that the Brewster's angle increases with $\tilde{\theta}$. At high frequencies another limit can be achieved: Keeping $\tilde{\theta}$ fixed at taking $n-1 \ll 1$ we have

$$
\tan \alpha_{B} \approx \frac{\tilde{\theta}}{2 \sqrt{n-1}} \sqrt{\tilde{\theta}^{2}+\sqrt{4+\tilde{\theta}^{4}}}
$$

In this case the Brewster's angle becomes close to $\pi / 2$. Since at large frequencies $n-1$ is proportional to $1 / \omega^{2}$, we derive that this limit as approached as $\pi / 2-\theta_{B} \propto 1 / \omega$.

\section{TRANSITION RADIATION}

The conventional transition radiation is emitted when a fast charged particle, i.e., a particle moving with energy much greater than the medium ionization energy, crosses the boundary between the two media having different dielectric constants [25-28]. Its main application is the tomographic investigation of the medium electromagnetic properties. The transition radiation originates from the difference of the photon wave function on the two sides of the boundary. In chiral matter with $\partial \theta \neq 0$ the photon dispersion relation is modified due to the chiral anomaly. As a result, when a fast charged particle crosses the boundary between the chiral matter and vacuum it emits the chiral transition radiation which was studied in Refs. [22,23]. In this section we argue that the chiral

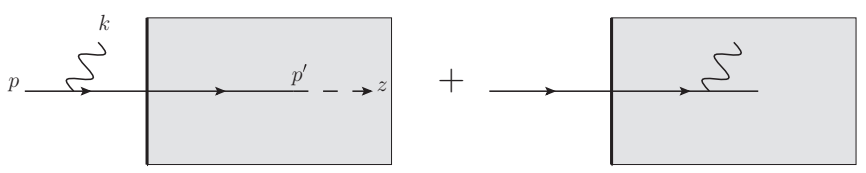

FIG. 3. Diagrams contributing to the photon radiation by a charged particle crossing the boundary. transition radiation is emitted even if $\theta$ is constant everywhere except at the boundary.

Consider a charged ultrarelativistic fermion moving along the $z$ axis as shown in Fig. 3. We assume that emitted photon energy $\omega$ is much larger than the plasma frequency of the medium $\omega_{\mathrm{pl}}$, which allows writing the photon dispersion relation as $\omega^{2}=\boldsymbol{k}^{2}+\omega_{\mathrm{pl}}^{2}$. The corresponding index of refraction is close to unity, $n-1=\omega_{\mathrm{pl}}^{2} / 2 \omega^{2}$. If the photon energy is so large that $n-1 \ll|\tilde{\theta}|$, one can neglect the deviation of the refractive index from unity. In this case the scattering amplitudes are given by Eqs. (12). The fact that they are independent of the photon momentum makes the calculation especially simple.

In the high-energy limit the wave function of the right-hand polarized photon, normalized to one particle per unit volume, reads

$$
\begin{aligned}
\boldsymbol{A}^{+}= & \frac{1}{\sqrt{2 \omega}}\left\{\left[\boldsymbol{\epsilon}_{i}^{+} e^{i \boldsymbol{k}_{i} \cdot \boldsymbol{r}}-\frac{i \tilde{\theta}}{2-i \tilde{\theta}} \boldsymbol{\epsilon}_{r}^{-} e^{i \boldsymbol{k}_{r} \cdot \boldsymbol{r}}\right] \eta(-z)\right. \\
& \left.+\frac{2}{2-i \tilde{\theta}} \boldsymbol{\epsilon}_{t}^{+} e^{i \boldsymbol{k}_{t} \cdot \boldsymbol{r}} \eta(z)\right\} e^{-i \omega t} \quad \text { if } \omega \gg \frac{\omega_{\mathrm{pl}}}{\sqrt{\tilde{\theta}}},
\end{aligned}
$$

where $\eta$ is a step function and the normalization volume is set to unity here and in (21). The wave function of the left-hand polarized photon $\boldsymbol{A}^{-}$can be obtained by complex conjugating the polarization vectors and the amplitudes in front of them. Let $\boldsymbol{k}_{\perp}$ denote the photon momentum perpendicular to the the fermion direction $\hat{z}$. Note that $k_{i x}=k_{r x}=k_{t x}=k_{\perp}$, as indicated in Sec. II. ${ }^{3}$ As for the longitudinal components, they are given by $k_{i z}=-k_{r z} \equiv k_{z}$ and $k_{t z}=\sqrt{n^{2} k_{z}^{2}+\left(n^{2}-1\right) k_{\perp}^{2}} \approx$ $k_{z}$. The quantization of the electromagnetic field in the presence of the semi-infinite dielectric material was discussed in Refs. [29,30].

The scattering matrix element for the photon emission at the leading order is

$$
S=-i e Q \int \bar{\psi}_{p^{\prime}} \gamma^{\mu} \psi_{p} A_{\mu}^{*} d^{4} x
$$

\footnotetext{
${ }^{3}$ The subscripts $\perp$ here and in Eqs. (4) are not to be confused.
} 
where $Q$ is the fermion electric charge in units of electron charge $e$ and $\psi_{p}$ is its wave function, which in the ultrarelativistic approximation reads

$$
\psi_{p}=\frac{1}{\sqrt{2 \varepsilon}} u(p) e^{i \varepsilon(z-t)} \exp \left\{i \boldsymbol{p}_{\perp} \cdot \boldsymbol{x}_{\perp}-i z \frac{\boldsymbol{p}_{\perp}^{2}+m^{2}}{2 \varepsilon}\right\},
$$

where $\varepsilon$ and $\boldsymbol{p}$ are the initial fermion energy and momentum, respectively, and $\boldsymbol{x}_{\perp}$ is a position vector in the $x y$ plane. The primed variables refer to the final fermion. In the reference frame that we chose $\boldsymbol{p}_{\perp}=0$. Taking integrals over time and $x_{\perp}$ one obtains the matrix element for the right-handed photon emission,

$$
S=-i e Q(2 \pi)^{3} \delta\left(\omega+\varepsilon^{\prime}-\varepsilon\right) \delta\left(\boldsymbol{k}_{\perp}+\boldsymbol{p}_{\perp}^{\prime}\right) \frac{\bar{u}\left(p^{\prime}\right) \notin^{*} u(p)}{\sqrt{8 \varepsilon \varepsilon^{\prime} \omega}} \int_{-\infty}^{\infty} d z e^{i\left(p_{z}-p_{z}^{\prime}\right) z}\left[\eta(-z)\left(e^{-i k_{z} z}-\frac{i \tilde{\theta}}{2-i \tilde{\theta}} e^{i k_{z} z}\right)+\eta(z) \frac{2}{2-i \tilde{\theta}} e^{-i k_{z} z}\right] .
$$

The longitudinal momentum transfer in the forward direction is small,

$$
p_{z}-p_{z}^{\prime}-k_{z} \approx-\frac{m^{2}}{2 \varepsilon}+\frac{p_{\perp}^{\prime 2}+m^{2}}{2 \varepsilon^{\prime}}+\frac{k_{\perp}^{2}+\omega_{\mathrm{pl}}^{2} \eta(z)}{2 \omega}=\frac{k_{\perp}^{2}+x^{2} m^{2}+(1-x) \omega_{\mathrm{pl}}^{2} \eta(z)}{2 \varepsilon x(1-x)}
$$

where $x=\omega / \varepsilon$. However, the longitudinal momentum transfer in the backward direction is very large, $p_{z}-p_{z}^{\prime}+k_{z} \approx 2 \omega$. Since the integrals in Eq. (22) are inversely proportional to the longitudinal momentum transfer, the reflected wave can be neglected in the high-energy limit. Thus the integrals in Eq. (22) yield

$$
\int_{-\infty}^{\infty} d z \cdots=-\frac{2 i \varepsilon x(1-x)}{k_{\perp}^{2}+x^{2} m^{2}}+\frac{2}{2-i \tilde{\theta}} \frac{2 i \varepsilon x(1-x)}{k_{\perp}^{2}+x^{2} m^{2}+(1-x) \omega_{\mathrm{pl}}^{2}} .
$$

Evidently, the two terms on the right-hand side correspond to the two diagrams in Fig. 3. The derivation of the photon spectrum from the scattering matrix follows the standard procedure with the result

$$
\frac{d N}{d^{2} k_{\perp} d x}=\frac{\alpha Q^{2}}{2 \pi^{2} x}\left\{\left(\frac{x^{2}}{2}-x+1\right) k_{\perp}^{2}+\frac{x^{4} m^{2}}{2}\right\} \sum_{ \pm}\left|\frac{1}{k_{\perp}^{2}+x^{2} m^{2}}-\frac{2}{2 \mp i \tilde{\theta}} \frac{1}{k_{\perp}^{2}+x^{2} m^{2}+(1-x) \omega_{\mathrm{pl}}^{2}}\right|^{2} .
$$

It is easy to see that the two polarizations contribute equally to the spectrum that reads

$$
\begin{aligned}
\frac{d N}{d^{2} k_{\perp} d x}= & \frac{\alpha Q^{2}}{\pi^{2} x}\left\{\left(\frac{x^{2}}{2}-x+1\right) k_{\perp}^{2}+\frac{x^{4} m^{2}}{2}\right\} \frac{1}{k_{\perp}^{2}+x^{2} m^{2}+(1-x) \omega_{\mathrm{pl}}^{2}}\left[\frac{(1-x)^{2} \omega_{\mathrm{pl}}^{4}}{\left(k_{\perp}^{2}+x^{2} m^{2}\right)^{2}\left[k_{\perp}^{2}+x^{2} m^{2}+(1-x) \omega_{\mathrm{pl}}^{2}\right]}\right. \\
& \left.+\frac{\tilde{\theta}^{2}}{4+\tilde{\theta}^{2}}\left(\frac{2}{k_{\perp}^{2}+x^{2} m^{2}+(1-x) \omega_{\mathrm{pl}}^{2}}-\frac{1}{k_{\perp}^{2}+x^{2} m^{2}}\right)\right] .
\end{aligned}
$$

In a particular case $\theta=0$ one obtains the usual formula for the transition radiation. The $\theta$-independent contribution in (26) falls off as $\omega_{\mathrm{pl}}^{4} / k_{\perp}^{6}$ with the photon transverse momentum, whereas the anomalous term only as $\tilde{\theta}^{2} / k_{\perp}^{2}$. We also note that the contribution of the gradients $\partial \theta$ to the transition radiation, calculated in Refs. [22,23], also decreases as $k_{\perp}^{-6}$. Therefore, the anomalous term in (26) dominates at large transverse momenta $k_{\perp} \gg \omega_{\mathrm{pl}} / \sqrt{\tilde{\theta}}$. This is illustrated in Fig. 4.

\section{SUMMARY}

The topological domains discussed in this paper are found in a variety of systems such as the Weyl semimetals and the quark-gluon plasma; the hypothetical axionic matter is possibly organized into such domains. At present little is known about their spacetime structure. The phenomenological models usually assume that the average topological charge density $\theta$ is a slow function of its space and time coordinates. It is reasonable to expect that in a finite-size domain the variation of $\theta$ is largest across the domain boundary, allowing one to neglect the variation of $\theta$ in the bulk.
Instead, it impacts the electromagnetic processes through the boundary conditions. This approximation is justified if the boundary width is negligible compared to the typical length scales. Since $\theta$ as a macroscopic field represents the

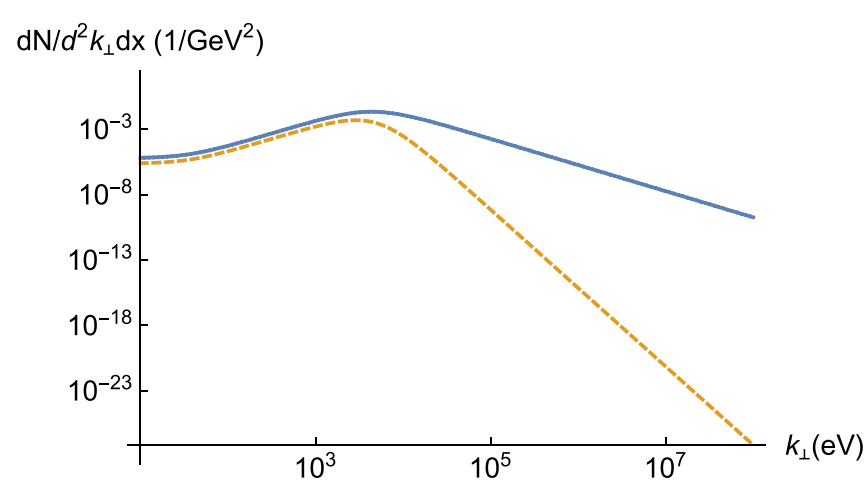

FIG. 4. Transition radiation spectrum for $Q=1, m=0.5 \mathrm{MeV}$ (electron), $\omega_{\mathrm{pl}}=10 \mathrm{eV}, x=10^{-2}, \tilde{\theta}=10^{-5}$. The solid line is the full spectrum, and the dashed line is its nonanomalous component. 
average over many microscopic topological domains, its boundary has a negligible width if the typical length scales are much larger than the interdomain distances. ${ }^{4}$ We used two examples to demonstrate the impact of $\theta$ on the physical observables and to propose possible avenues for experimental investigation.

In the first example, we considered the refraction of the electromagnetic wave on a flat surface of a domain with finite $\theta$. We derived the modified Fresnel equations (10) for the amplitudes of reflected and transmitted waves. At high frequencies, when the refraction index is close to unity, there is still "topological" refraction due only to the finite value of $\theta$. In this case, the amplitudes of the transmitted and reflected waves do not depend on the angle of incidence and it is the circular polarization that is preserved (not the linear one as in the conventional case $\theta=0$ ). One can measure the value of $\tilde{\theta}$ by observing the Brewster's angle as shown in Fig. 2.

In the second example, we derived the spectrum of transition radiation for an ultrarelativistic particle crossing the boundary between the vacuum and the finite- $\theta$ domain. We found that at high transverse momenta, photon radiation due to the chiral anomaly dominates over the conventional

\footnotetext{
${ }^{4}$ The quark-gluon plasma produced in heavy-ion collisions may contain a few topological domains that have sizes larger or comparable to the typical length scales. The extent of applicability of our results to that physical system depends on the spacetime structure of the domains at finite temperature, viz., whether $\theta$ has a sharp or smooth boundary.
}

transition radiation mechanism. Moreover, it also dominates over the chiral transition radiation emitted due to the finite time derivative of $\theta$. Thus, this represents an opportunity to establish the existence of the $C P$-odd domains of constant topological charge density and measure the magnitude of $\theta$.

\section{ACKNOWLEDGMENT}

This work was supported in part by the U.S. Department of Energy under Grant No. DE-FG02-87ER40371.

\section{APPENDIX: REFRACTION AT A THIN FILM}

If a right-hand polarized wave is normally incident at a thin film of width $d$, then by following the prescription of Sec. II we obtain for the first boundary

$$
\begin{aligned}
& A_{i}^{+}+A_{r}^{-}=a_{t}^{+}+a_{r}^{-}, \\
& A_{i}^{+}-A_{r}^{-}=a_{t}^{+}(n-i \tilde{\theta} n)+a_{r}^{-}(n+i \tilde{\theta} n),
\end{aligned}
$$

where $a_{r}^{-}$and $a_{t}^{+}$denote the amplitudes of the left- and rightpropagating waves inside the film.

For the second boundary we obtain

$$
\begin{aligned}
a_{t}^{+} \exp (i d \omega n)+a_{r}^{-} \exp (-i d \omega n) & =A_{t}^{+} \exp (i \omega d), \\
a_{t}^{+}(n-i \tilde{\theta} n) \exp (i d \omega n)+a_{r}^{-}(n+i \tilde{\theta} n) \exp (-i d \omega n) & =A_{t}^{+} \exp (i \omega d) .
\end{aligned}
$$

The amplitudes of the nonvanishing transmitted and reflected waves are

$$
\begin{aligned}
A_{t}^{+} & =\frac{4 n \exp [i d \omega(n-1)]}{1+n\left(2+n+n \tilde{\theta}^{2}\right)-\exp (2 i d \omega n)\left[1+n\left(-2+n+n \tilde{\theta}^{2}\right)\right]} A_{i}^{+}, \\
A_{r}^{-} & =\frac{\left[1-n\left(n-2 i \tilde{\theta}+n \tilde{\theta}^{2}\right)\right] \sin (d \omega n)}{2 i n \cos (d \omega n)+\left[1+n^{2}\left(1+\tilde{\theta}^{2}\right)\right] \sin (d \omega n)} A_{i}^{+} .
\end{aligned}
$$

At $\theta=0$ these equations reduce to the known result [31]. At high frequencies $n=1$ and Eqs. (A3) reduce to

$$
\begin{aligned}
A_{t}^{+} & =\frac{4}{4+\tilde{\theta}^{2}[1-\exp (2 i d \omega)]} A_{i}^{+}, \\
A_{r}^{-} & =\frac{\tilde{\theta}(\tilde{\theta}-2 i) \sin (d \omega)}{2 i \cos (d \omega)+\left(2+\tilde{\theta}^{2}\right) \sin (d \omega)} A_{i}^{+} .
\end{aligned}
$$

[1] S. L. Adler, Axial vector vertex in spinor electrodynamics, Phys. Rev. 177, 2426 (1969).

[2] J. S. Bell and R. Jackiw, A PCAC puzzle: $\pi_{0} \rightarrow \gamma \gamma$ in the sigma model, Nuovo Cimento A 60, 47 (1969).

[3] D. E. Kharzeev, J. Liao, S. A. Voloshin, and G. Wang, Chiral magnetic and vortical effects in high-energy nuclear collisions-A status report, Prog. Part. Nucl. Phys. 88, 1 (2016).
[4] A. R. Zhitnitsky, $P$-odd fluctuations and long range order in heavy ion collisions. Deformed QCD as a toy model, Nucl. Phys. A 897, 93 (2013).

[5] D. Kharzeev and A. Zhitnitsky, Charge separation induced by $P$-odd bubbles in QCD matter, Nucl. Phys. A 797, 67 (2007).

[6] Q. Li et al., Observation of the chiral magnetic effect in $\mathrm{ZrTe}_{5}$, Nat. Phys. 12, 550 (2016). 
[7] D. J. E. Marsh, Axion cosmology, Phys. Rep. 643, 1 (2016).

[8] F. Wilczek, Two Applications of Axion Electrodynamics, Phys. Rev. Lett. 58, 1799 (1987).

[9] S. M. Carroll, G. B. Field, and R. Jackiw, Limits on a Lorentz and parity violating modification of electrodynamics, Phys. Rev. D 41, 1231 (1990).

[10] P. Sikivie, On the interaction of magnetic monopoles with axionic domain walls, Phys. Lett. B 137, 353 (1984).

[11] K. Fukushima, D. E. Kharzeev, and H. J. Warringa, The chiral magnetic effect, Phys. Rev. D 78, 074033 (2008).

[12] D. E. Kharzeev, Topologically induced local $P$ and $C P$ violation in QCD $\times$ QED, Ann. Phys. 325, 205 (2010).

[13] D. E. Kharzeev and H. J. Warringa, Chiral magnetic conductivity, Phys. Rev. D 80, 034028 (2009).

[14] A. A. Burkov, Chiral anomaly and transport in Weyl metals, J. Phys.: Condens. Matter 27, 113201 (2015).

[15] D. E. Kharzeev, The chiral magnetic effect and anomalyinduced transport, Prog. Part. Nucl. Phys. 75, 133 (2014).

[16] D. Kharzeev, Parity violation in hot QCD: Why it can happen, and how to look for it, Phys. Lett. B 633, 260 (2006).

[17] D. E. Kharzeev, L. D. McLerran, and H. J. Warringa, The effects of topological charge change in heavy ion collisions: Event by event $P$ and $C P$ violation, Nucl. Phys. A 803, 227 (2008).

[18] M. Joyce and M. E. Shaposhnikov, Primordial Magnetic Fields, Right-Handed Electrons, and the Abelian Anomaly, Phys. Rev. Lett. 79, 1193 (1997).

[19] A. Boyarsky, J. Frohlich, and O. Ruchayskiy, Self-Consistent Evolution of Magnetic Fields and Chiral Asymmetry in the Early Universe, Phys. Rev. Lett. 108, 031301 (2012).
[20] Y. Akamatsu and N. Yamamoto, Chiral Plasma Instabilities, Phys. Rev. Lett. 111, 052002 (2013).

[21] K. Tuchin, Impact of domain walls on the chiral magnetic effect in hot QCD matter, Phys. Rev. C 97, 064914 (2018).

[22] K. Tuchin, Radiative instability of quantum electrodynamics in chiral matter, Phys. Lett. B 786, 249 (2018).

[23] X. G. Huang and K. Tuchin, Transition Radiation as a Probe of the Chiral Anomaly, Phys. Rev. Lett. 121, 182301 (2018).

[24] T. Hayata, Chiral magnetic effect of light, Phys. Rev. B 97, 205102 (2018).

[25] V. L. Ginzburg and I. M. Frank, Radiation of a uniformly moving electron due to its transition from one medium into another, J. Phys. (USSR) 9, 353 (1945) [Zh. Eksp. Teor. Fiz. 16, 15 (1946)].

[26] V. L. Ginzburg and V. N. Tsytovich, Several problems of the theory of transition radiation and transition scattering, Phys. Rep. 49, 1 (1979).

[27] V. N. Baier and V. M. Katkov, Quantum theory of transition radiation and transition pair creation, Phys. Lett. A 252, 263 (1999).

[28] D. Schildknecht and B. G. Zakharov, Transition radiation in quantum regime as a diffractive phenomenon, Phys. Lett. A 355, 289 (2006).

[29] C. K. Carniglia and L. Mandel, Quantization of evanescent electromagnetic waves, Phys. Rev. D 3, 280 (1971).

[30] I. Bialynicki-Birula and J. B. Brojan, Completeness of evanescent waves, Phys. Rev. D 5, 485 (1972).

[31] M. Born and E. Wolf, Principles of Optics, 7th ed. (Cambridge University Press, Cambridge, U.K., 1999), p. 65. 